\title{
Precipitation of oxide phases in titanium alloys with rare earth metals
}

\author{
M.A. Popova ${ }^{\dagger}$, N.G. Rossina, N.A. Popov, M.O. Leder \\ †m.a.popova@urfu.ru
}

Ural Federal University, 19 Mira St., 620002, Yekaterinburg, Russia

\begin{abstract}
The effect of gadolinium microalloying on the formation of oxide and intermetallic phases in heat-resistant pseudo-alpha and two-phase titanium alloys of different chemical compositions during the crystallization and subsequent heat treatment was studied. By means of scanning electron microscopy it was shown that in the alloy containing tin an intermetallic compound of a $\mathrm{Gd}_{x} \mathrm{Sn}_{y}$ system was formed during crystallization. Gadolinium oxide was subsequently formed on the particles of this intermetallic compound. As a result, these particles have a complex internal structure. The nucleation of these particles occurs primarily in the body of a grain at interfaces. Annealing in beta-phase and two-phase regions with a change in morphology of alpha and beta phases does not lead to a change in the location of such particles. The nature of their distribution allows one to state that they do not significantly affect the properties of the alloy. In the alloys without tin, gadolinium oxide was formed during cooling primarily at the grain and interphase boundaries. The size of such particles is substantially less than that in alloys with tin, while the amount is greater. The heat treatment in the beta-region (homogenization annealing) is accompanied by an increase of the number and size of particles both on grain boundaries and within the grains. Moreover, the gadolinium concentration in solid solution is slightly higher than that in alloys with tin. The particles precipitated at grain boundaries are assumed to decrease the viscous and plastic properties leading to their reduction.
\end{abstract}

Keywords: titanium alloys, rare earth metals, oxide phase, SEM.

\section{Выделение оксидных фаз в титановых сплавах с редкоземельными элементами}

\author{
Попова М.А. ${ }^{\dagger}$ Россина Н.Г., Попов Н.А., Ледер М.О. \\ †m.a.popova@urfu.ru
}

Уральский Федеральный Университет, ул. Мира 19, 620002, Екатеринбург, Россия

\begin{abstract}
Изучено влияние микролегирования редкоземельным элементом - гадолинием на образование интерметаллидных и оксидных фаз в жаропрочных псевдо-альфа и двухфазных титановых сплавах с различным химическим составом при кристаллизации и последующей термической обработке. Методами микрорентгеноспектрального анализа показано, что в сплавах, содержащих легкоплавкий элемент - олово, при кристаллизации формируется интерметаллид системы $\mathrm{Gd}_{x} \mathrm{Sn}_{y}$, на котором в дальнейшем образуется оксид гадолиния, обволакивая исходную частицу интерметаллида. В результате такие частицы имеют сложное внутреннее строение. Зарождение их в основном происходит в теле зерен по межфазным поверхностям раздела. Проведение отжига как в однофазной бета-области, так и в двухфазной области, изменяя морфологию альфа- и бета-фаз, не меняет расположение таких частиц. Характер их распределения позволяет утверждать, что они не должны оказывать существенного влияния на свойства сплава. В тех случаях, когда в сплаве нет олова, при охлаждении в процессе кристаллизации формируется оксид гадолиния преимущественно на межзеренных и межфазных границах раздела. Размер таких частиц существенно меньше, чем в сплавах с оловом, а количество больше. Термическая обработка в бета-области (гомогенизационный отжиг) сопровождается увеличением количества и размеров частиц, как по границам зерен, так и теле зерна. При этом концентрация гадолиния в твердом растворе несколько больше, чем в сплавах с оловом. Выделившиеся по границам раздела частицы должны оказывать влияние на вязкостные и пластические свойства понижая их.
\end{abstract}

Ключевые слова: титановые сплавы, редкоземельные металлы, оксидные фазы, редкоземельные элементы. 


\section{1. Введение}

Применение редкоземельных элементов (РЗЭ) в качестве микродобавок в конструкционные и жаропрочные сплавы, по мнению многих исследователей $[1,2]$, должно способствовать получению более стабильных структурных состояний и, тем самым, более стабильных свойств. Это влияние обусловлено несколькими факторами. Во-первых, РЗЭ являются эффективными рафинирующими добавками, которые нейтрализуют влияние таких элементов внедрения как кислород, связывая их в оксиды и, тем самым, уменьшают концентрацию кислорода на границах раздела [3]. В результате должны повышаться характеристики вязкости и пластичности материала. Во-вторых, РЗЭ являются поверхностно-активными элементами, и поэтому их концентрация на границах раздела должна быть выше средней. Как следствие этого, подвижность границ будет пониженной, что обусловит повышенную термическую стабильность сплава [4]. При этом есть данные о том, что микролегирование уменьшает ликвационную неоднородность и препятствует образованию вредных фаз [2]. Как утверждается в некоторых работах, микролегирование РЗЭ способствует измельчению зерен [3]. Считается, что одной из возможностей увеличения рабочих температур титановых сплавов является их микролегирование РЗЭ. Повышение жаростойкости и термической стабильности обусловлено образованием термостойких окисных пленок на основе РЗЭ, имеющих хорошую адгезию к основному металлу [5]. Однако до настоящего времени механизм влияния добавок РЗЭ на структуру и свойства титановых сплавов остается не установленным.

Как правило, для титановых сплавов в качестве РЗЭ используются церий [6], иттрий [7], неодим [8] и гадолиний [9]. Поскольку количество вводимого элемента не превышает 0,2 масс. \%, возникают технические сложности в определении характера расположения атомов вводимого РЗЭ и его концентрации в твердом растворе. В этой связи в данной работе рассмотрено влияние повышенного относительно обычного содержания Р3Э в сплавах различных систем легирования для выяснения характера распределения элемента между фазами сплава. В качестве РЗЭ был выбран гадолиний, который относится к иттриевой подгруппе и имеет ограниченную растворимость в $\alpha$-титане [10]. Данных по влиянию гадолиния на свойства сплавов титана относительно мало $[9,11]$, в то время как работ, посвященных влиянию иттрия довольно много, например, $[7,12,13]$. Учитывая принадлежность обоих элементов к одной подгруппе, следует ожидать схожесть тенденций по влиянию этих элементов на свойства сплавов. Присутствие иттрия около границ зерен способствует закреплению вредных примесей в комплексы с ним, что приводит к рафинированию матрицы, повышая ее эксплуатационные свойства. Целью настоящей работы явилось изучение влияния микролегирования гадолинием в количестве до 0,4 масс. \% на микроструктуру жаропрочных титановых сплавов на основе систем Ti-Al-Mo-Zr-Si (Сплав 1) и Ti-Al-Mo-Zr-Sn-Si (Сплав 2).

\section{2. Материалы и методы}

Слитки, массой 20 кг, получали методом двойного вакуумного переплава. Последующую деформацию осуществляли комбинированной ковкой и прокаткой с окончанием температуры деформации в $\beta$-области. Термическую обработку проводили в электрических муфельных печах при $T=1100 \ldots 1250^{\circ} \mathrm{C}$. Образцы для исследований вырезали из центральных участков слитка. Анализ микроструктуры проводили с помощью растрового электронного микроскопа Jeol JSM 6490 LV и электронно-ионного микроскопа CrossBeam Auriga Carl Zeiss. В случае локального определения состава методом МРСА анализируемая точка соответствует верхнему левому углу надписи «Spectrum».

\section{3. Результаты}

На рис.1 представлена микроструктура сплава 1 в литом состоянии.

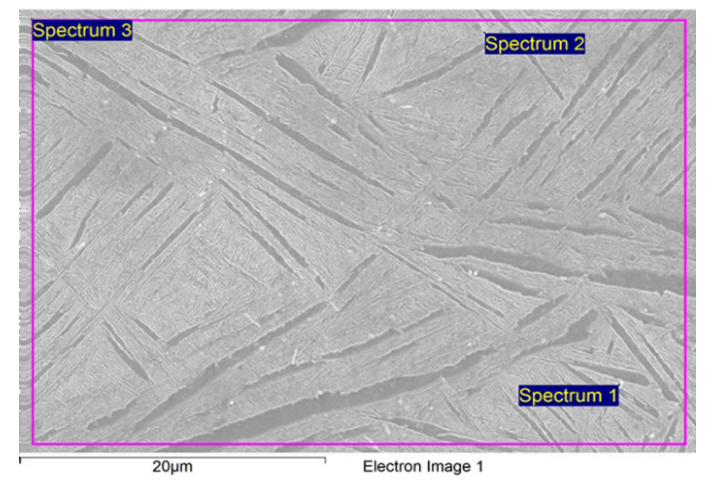

\begin{tabular}{|c|c|c|c|c|c|c|}
\hline Spectrum & $\mathrm{O}$ & $\mathrm{Al}$ & $\mathrm{Ti}$ & $\mathrm{Zr}$ & $\mathrm{Mo}$ & $\mathrm{Gd}$ \\
\hline Spectrum 1 & 13.3 & 4.4 & 49.8 & 0.0 & 2.5 & 30.0 \\
\hline Spectrum 2 & 17.2 & 4.2 & 49.0 & 0.0 & 2.6 & 27.0 \\
\hline Spectrum 3 & 0.00 & 6.1 & 87.2 & 1.3 & 4.4 & 1.0 \\
\hline
\end{tabular}

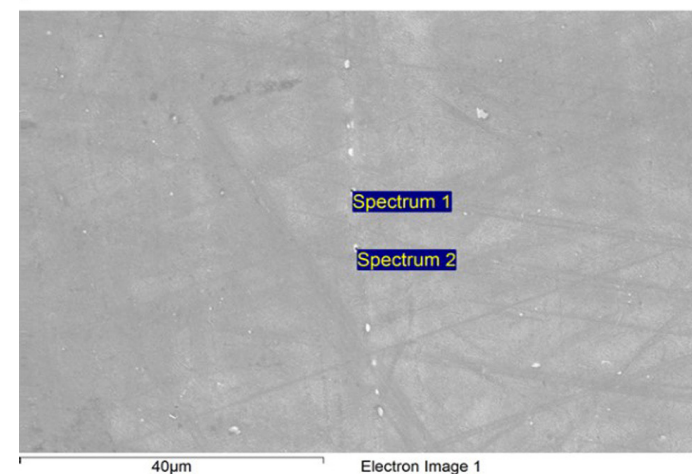

\begin{tabular}{|c|c|c|c|c|c|c|c|}
\hline Spectrum & $\mathrm{O}$ & $\mathrm{Al}$ & $\mathrm{Si}$ & $\mathrm{Ti}$ & $\mathrm{Zr}$ & $\mathrm{Mo}$ & $\mathrm{Gd}$ \\
\hline Spectrum 1 & 15.7 & 3.4 & 0.0 & 29.4 & 1.9 & 2.6 & 47.0 \\
\hline Spectrum 2 & 16.9 & 3.8 & 0.7 & 35.9 & 1.5 & 2.0 & 39.2 \\
\hline
\end{tabular}

Рис. 1. Микроструктура сплава системы Ti-Al-Mo-Zr-Si в литом состоянии.

Fig. 1. SEM images showing the as-cast alloy microstructure of the Ti-Al-Mo-Zr-Si system. 
Как видно из представленных фотографий, сплав имеет пластинчатую $(\alpha+\beta)$-структуру. Как внутри $\beta$-зерен, так и по границам наблюдаются относительно дисперсные выделения. Выделения в теле $\beta$-зерен преимущественно расположены на межфазных $\alpha / \beta$-границах. Анализ таких частиц методами микрорентгеноспектрального анализа показал, что они представляют собой оксиды гадолиния. Термическая обработка в $\beta$-области $\left(T=1100^{\circ} \mathrm{C}, \tau=1\right.$ час, охлаждение на воздухе) привела к увеличению количества и размера выделений оксида гадолиния по границам $\beta$-зерен (рис. 2). Размер частиц

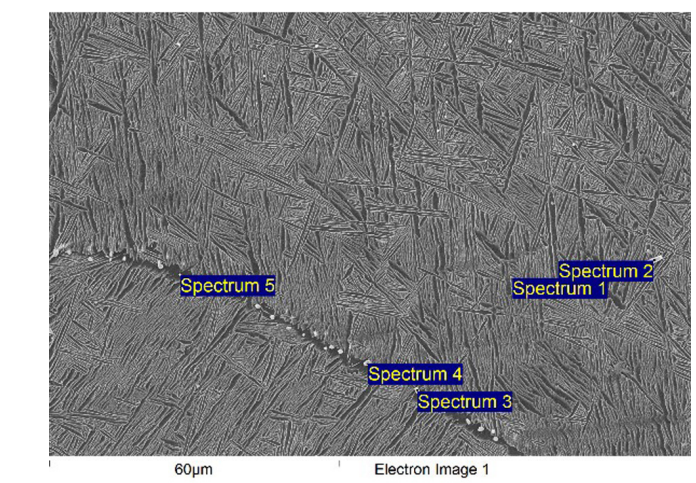

\begin{tabular}{|c|c|c|c|c|c|c|c|}
\hline Spectrum & $\mathrm{O}$ & $\mathrm{Al}$ & $\mathrm{Si}$ & $\mathrm{Ti}$ & $\mathrm{Zr}$ & $\mathrm{Mo}$ & $\mathrm{Gd}$ \\
\hline Spectrum 1 & 13.6 & 1.0 & 0.3 & 19.9 & 0.00 & 5.4 & 59.8 \\
\hline Spectrum 2 & 14.1 & 0.7 & 0.0 & 11.9 & 0.00 & 1.9 & 71.4 \\
\hline Spectrum 3 & 16.3 & 3.0 & 0.2 & 34.8 & 0.7 & 2.0 & 43.0 \\
\hline Spectrum 4 & 16.8 & 1.9 & 0.2 & 29.1 & 1.0 & 1.7 & 49.3 \\
\hline Spectrum 5 & & 6.0 & 0.5 & 88.0 & 1.3 & 4.2 & 0.0 \\
\hline
\end{tabular}

a

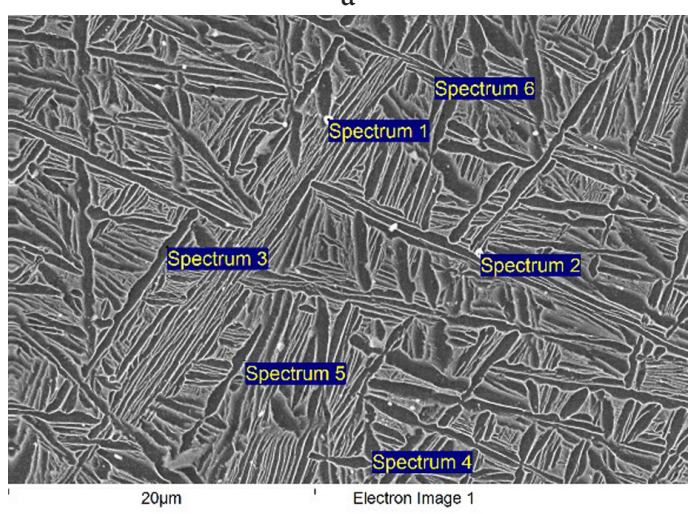

\begin{tabular}{|c|c|c|c|c|c|c|c|}
\hline Spectrum & $\mathrm{O}$ & $\mathrm{Al}$ & $\mathrm{Si}$ & $\mathrm{Ti}$ & $\mathrm{Zr}$ & $\mathrm{Mo}$ & $\mathrm{Gd}$ \\
\hline Spectrum 1 & 16.4 & 3.9 & 0.1 & 43.1 & 1.2 & 2.9 & 32.4 \\
\hline Spectrum 2 & 15.3 & 3.9 & 0.3 & 41.0 & 0.8 & 2.3 & 36.4 \\
\hline Spectrum 3 & & 5.8 & 0.3 & 89.1 & 1.1 & 3.3 & 0.4 \\
\hline Spectrum 4 & & 6.1 & 0.2 & 88.2 & 1.9 & 3.6 & 0.0 \\
\hline Spectrum 5 & & 6.3 & 0.3 & 85.6 & 1.7 & 6.1 & 0.0 \\
\hline Spectrum 6 & & 6.0 & 0.3 & 87.5 & 1.7 & 4.5 & 0.0 \\
\hline
\end{tabular}

$\mathrm{b}$

Pис. 2. Микроструктура сплава системы Ti-Al-Mo-Zr-Si в термообработанном состоянии.

Fig. 2. SEM images showing the heat-treated alloy microstructure of the Ti-Al-Mo-Zr-Si system. по границам составляет 1,5...2,0 мкм. При этом выделения в теле $\beta$-зерен также увеличиваются (рисунок $2 \mathrm{~b}$ ), но их размер несколько меньше, чем по границам зерен и составляет $0,7 \ldots 1,0$ мкм.

Полученные результаты не позволяют однозначно судить о растворении атомов гадолиния в $\alpha$-твердом растворе. Как правило, чувствительности прибора недостаточно для оценки его количества. Однако, как показывает «Спектр 3» на рис. 2b, его концентрация в $\alpha$-твердом растворе может достигать 0,4 масс. \%.

Для сплава 2 структура сплава также пластинчатая, но наблюдаемые выделения преимущественно располагаются по телу $\beta$-зерен (рис. 3). Выделений по границам зерен не обнаружено. Кроме того, образовавшиеся выделения имеют сложное строение. По данным микрорентгеноспектраль-ного анализа такие частицы содержат как гадолиний и кислород, так и олово. Используя метод ионной резки непосредственно в колонне микроскопа, удалось установить, что центральная часть таких частиц представляет собой интерметаллид, основными компонентами которого являются гадолиний и олово, а поверхностный слой - оксид гадолиния.
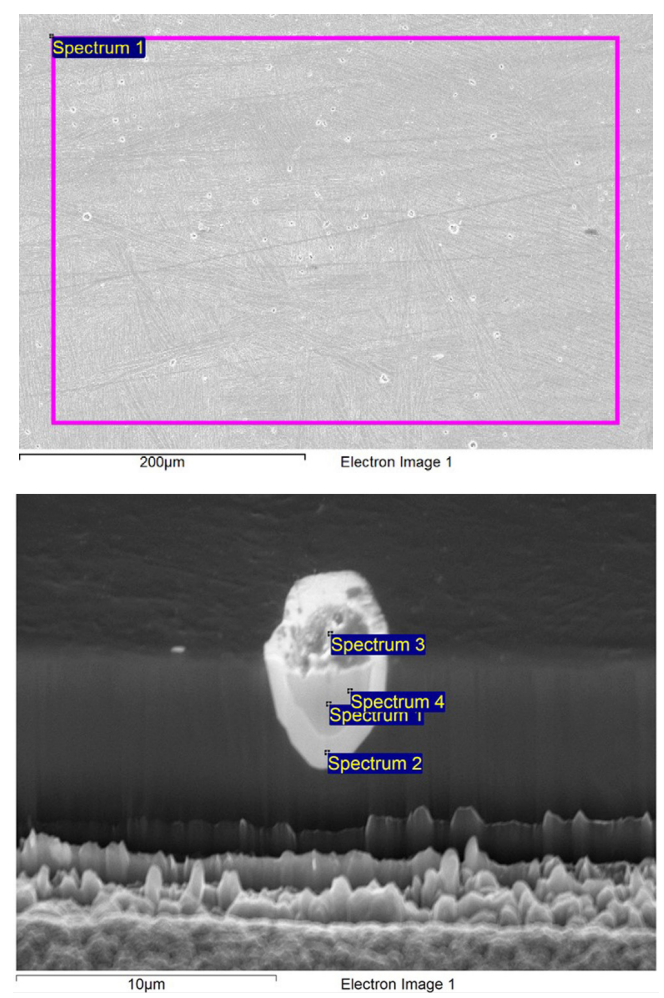

\begin{tabular}{|c|c|c|c|c|c|c|c|c|}
\hline Spectrum & $\mathrm{O}$ & $\mathrm{Al}$ & $\mathrm{Ti}$ & $\mathrm{Zr}$ & $\mathrm{Nb}$ & $\mathrm{Mo}$ & $\mathrm{Sn}$ & $\mathrm{Gd}$ \\
\hline Spectrum 1 & 2.1 & 1.2 & 14.8 & 1.3 & 0.6 & 0.1 & 23.9 & 56.0 \\
\hline Spectrum 2 & 8.6 & 1.5 & 23.4 & 2.0 & & & 2.1 & 62.4 \\
\hline Spectrum 3 & 22.6 & & 2.9 & 0.0 & & & 25.0 & 49.5 \\
\hline Spectrum 4 & 3.2 & 1.0 & 13.5 & 1.8 & & 0.2 & 24.8 & 55.5 \\
\hline
\end{tabular}

All results in weight \%

Рис. 3. Микроструктура сплава системы Ti-Al-Mo-Zr-Sn-Si в литом состоянии.

Fig. 3. SEM images of the as-cast alloy microstructure in the Ti-AlMo-Zr-Sn-Si system. 
По данным микрорентгеноспектрального анализа сложно однозначно утверждать о кристаллической структуре образующегося интерметаллида, но большинство проведенных измерений свидетельствует о том, что соотношение атомов гадолиния и олова в нем близко к 5:3. Следовательно, кристаллическая структура этого интерметаллида описывается как $\mathrm{Gd}_{5} \mathrm{Sn}_{3}$, и он относится к структурному типу $\mathrm{Mn}_{5} \mathrm{Si}_{3}$ с гексагональной решеткой при отношении осей $c / a \sim 0,7$. Для жаропрочных титановых сплавов характерно выделение силицидных частиц этого типа - (Ti,Zr) $)_{5} \mathrm{Si}_{3}[14,15]$. Такие частицы преимущественно выделяются на межфазных границах раздела и способствуют охрупчиванию сплавов [16]. По всей видимости следует ожидать, что и в сплавах с гадолинием этот эффект может иметь место. Поэтому следует избегать образования подобных выделений так как, во-первых, при их образовании уменьшается концентрация олова в твердом растворе, которое благоприятно влияет на жаропрочность и увеличивает прокаливаемость сплавов, а, во-вторых, сплав с такими частицами будет иметь пониженные характеристики вязкости и пластичности при комнатной температуре.

Проведение термической обработки в двухфазной области позволило сформировать преимущественно глобулярную структуру (рис. 4). Гадолиний по-прежнему в основном находится в частицах Gd-Sn-O, однако небольшое его количество (около $0,2 \ldots 0,3$ масс. \%) имеется и в твердом растворе. При повышении температуры обработки сплава выше температуры полиморфного превращения до $T=1100^{\circ} \mathrm{C}$ характер распределения частиц и их размер практически не меняется. Формирующаяся при охлаждении пластинчатая структура не связана с местами выделения этих частиц и, следовательно, они не должны оказывать существенного влияния на свойства сплава и его термическую стабильность.

\section{4. Выводы}

Полученные результаты позволяют утверждать, что если в сплаве присутствует олово, то формирующиеся частицы имеют сложное строение. Оксидная фаза $\left(\mathrm{Gd}_{x} \mathrm{O}_{y}\right)$ формируется на частицах интерметаллида $\mathrm{Gd}_{5} \mathrm{Sn}_{3}$. Ха-

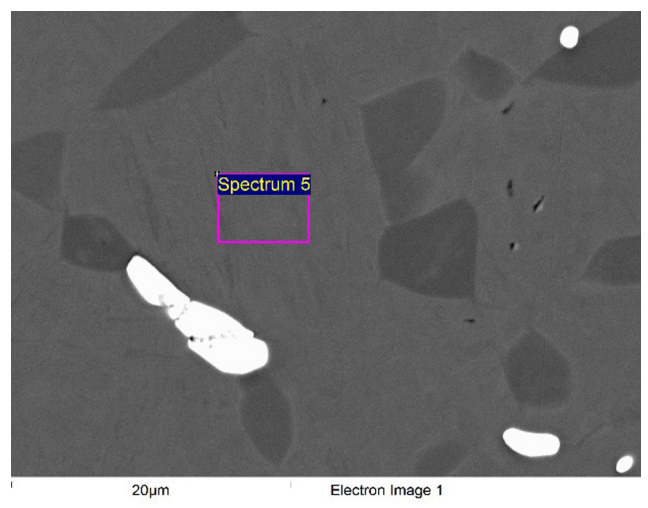

Pис. 4. Микроструктура сплава системы Ti-Al-Mo-Zr-Sn-Si после термообработки в двухфазной области.

Fig. 4. SEM image of the Ti-Al-Mo-Zr-Sn-Si alloy microstructure after the heat treatment in the two-phase field. рактер ее распределения позволяет утверждать, что она не должна оказывать существенного влияния на свойства сплава. В тоже время в сплаве без олова оксид гадолиния выделяется по межзеренным и межфазным границам и, тем самым, может оказать влияние на его механические свойства.

Благодарность/Aknowledgements. Работа выполнена при финансовой поддержке Министерства образования и науки Российской Федерации в рамках субсидирования соглашения от 29 сентября 2014 2. (№. 14.581.21.0002, уникальный идентификатор соглашения RFMEFI58114Х0002) Федеральной иелевой программы «Исследования и разработки по приоритетным направлениям развития научно-технологического комплекса России на 2014-2020 годь»».

\section{Литература/References}

1. A. I. Khorev. Russ. Eng. Research. 31, $1087-1094$ (2011), doi:10.3103/S1068798X11110104

2. Junko Hieda. Mater. Trans. 54, $1361-1367$ (2013), doi:10.2320/matertrans.MF201308

3. Hanguang Fu. Mater. Science and Engineering A 395, 281 - 287 (2005), doi:10.1016/j.msea.2004.12.029

4. Mingyue Zhao. J. of Refractory Metals and Hard Materials 48, 19-23 (2015), doi:10.1016/j.ijrmhm.2014.07.014

5. V.A. Kaschuk, M. B. Svetlov, The effect of small additions of rare earth metals and rhenium on the properties of titanium alloy VT5L, Science. Thought, Kiev 1975, $85-91$

6. N. M. Ulyakova. Met. Sci. \& Heat treatment, 3, 30-31 (1994).

7. S. R. Seagle, C.S. Hall, H.B. Romberger. Proc. of the 4th International Conf. on Titanium. New York. (1980). V.3. P. $2169-2175$.

8. G. P. Li, D. Li, Y. Y. Liu, S. X. Guan, Q. J. Wang, D. H. Ping, and Z.Q. Hu. Metallurgical and materials transactions A, 28A, (1997), doi:10.1007/s11661-997-0251-y

9. M. Holm, T. Ebel, M. Dahms. Materials and Design, 51, 943 - 948 (2013), doi:10.1016/j.matdes.2013.05.003

10. N. P. Lyakishev. Diagrams of Binary Metallic Systems. A Handbook. Moscow, Mechanical engineering. (1996), 872 p. (in Russian). [Н. П. Лякишев. Диаграммы состояния двойных металлических систем. Справочник. М.: Машиностроение. 1996. 872 с.]

11. N.A. Nochovnaya, A.I. Khorev, A.L. Yakovlev. Metal Science and Heat Treatment, 55, 415-419 (2013), doi:10.1007/s11041-013-9646-0

12. L. Zeng, Q. Hong, Y. Zhao, Y. Qi. Rare Met. Mater. \& Eng. 43, 2407-2410 (2014).

13. W. F. Cui, C. M. Liu, L. Zhou, G. Z. Luo. Mater. Sci. \& Eng. A. 323, $192-197$ (2002).

14. A.A. Popov, N.A. Drozdova. Physics of Metals and Metallography. 84, 407-412 (1997).

15. C. Ramachandra, V. Singh, Met. Trans., A. 23, 689-690 (1992).

16. A. A. Popov, M. O. Leder, M.A. Popova, N. G. Rossina, I. V. Narygina. Physics of Metals and Metallography. 116, 3261 - 266 (2015), doi:10.1134/S0031918X15030102 